\title{
Evaluation of the social, clinical and laboratorial profile of patients diagnosed with leprosy in a reference center in São Paulo*
}

\author{
Ana Carolina Souza Porto ${ }^{1}$ \\ Jaison Antônio Barreto ${ }^{2}$ \\ Renata Borges Fortes Costa Figueira ${ }^{2}$ \\ José Roberto Pereira Lauris ${ }^{3}$
}

DOI: http://dx.doi.org/10.1590/abd1806-4841.20153385

\begin{abstract}
BACKGROUND: Leprosy is the main infectious cause of disability. It is said to be eradicated in São Paulo since 2005, but diagnosis is still late.

OBjeCTIVES: To investigate the social, clinical and laboratorial profile of leprosy patients diagnosed between 01/2007 and 12/2011, in a reference center in São Paulo.

METHODS: Retrospective descriptive study. Data of all new leprosy cases diagnosed between 01/2007 and 12/2011 were raised in São Paulo.

RESULTS: 103 men and 71 women were diagnosed, most of them were multibacillary. Mean age at diagnosis was 49 yrs; 2,2\% were children; $70 \%$ had incomplete primary education; $50 \%$ were referred without diagnostic suspicion of leprosy. Mean time since first symptoms/signs and diagnosis was 2 years; $64 \%$ of patients had some degree of disability, and $26 \%$ had grade 2.23 cases were diagnosed only after being summoned, and $80 \%$ of these had no disability. Agreement between the Ridley and Jopling and the WHO classification was 75\% (kappa index $=0.44$ ). Serology for IgM anti-PGL1 (87 patients) showed a mean value of 0.25 , and an association between MB classification and test positivity $(\mathrm{p}<0.001)$.

ConCLUSIONS: Leprosy diagnosis in São Paulo is late. The disease mainly affected the socially disadvantaged and economically active population. Failure to detect the disease ( $41 \%$ in the last 10 years) could be due to the lack of suspicion and to decentralization. For the classification of patients with advanced leprosy, both the WHO and R\&J classifications proved to be helpful tools. Keywords: Epidemiology; Leprosy; Mycobacterium leprae; Public health
\end{abstract}

\section{INTRODUCTION}

Leprosy is a primarily dermatological, granulomatous infectious disease caused by the obligate intracellular organism Mycobacterium leprae. ${ }^{1,2}$ It is one of the oldest diseases that affect humans, with references dating back to $600 \mathrm{BC}$. Although the etiologic agent was identified in 1873 by the Norwegian scientist Amauer Hansen, it is still the leading infectious cause of disability. Leprosy can lead to deformities that stigmatize those affected by it. This perpetuates theirs and their families' social disadvantages. $1,2,3,4$

In 1991, the World Health Organization (WHO) set a target of eliminating leprosy as a public health problem, which is defined as a prevalence lower than 1:10,000 inhabitants. ${ }^{1}$ After the large-scale introduction of polychemotherapy (PCT) - a highly effective thera- peutic regimen - in 1981, the concept of "discharge by cure" was defined. All cases which were in the active record at that time were reclassified, and those patients who had active disease were treated and subsequently removed from prevalence records. ${ }^{1,45}$ This resulted in a significant reduction in prevalence, and the target of eliminating leprosy as a problem health was achieved globally in 2000. Treatment time for multibacillary (MB) cases was reduced from two years to one, with no scientific evidence to justify this change. ${ }^{6}$ However, the detection rate of new cases remained high in the last 20 years and disease transmission goes on. ${ }^{1}$ Thus, prevalence rate is vulnerable to operational factors, and is not suitable to be usead as an indicator of disease control. ${ }^{1,5}$ Other indicators for surveillance of leprosy are

Approved by the Advisory Board and accepted for publication on 16.05.2014.

* Study conducted at the Instituto Lauro de Souza Lima (ILSL) - Bauru (SP), Brazil.

Financial Support: None

Conflict of Interest: None.

Universidade Federal de São Paulo (UNIFESP) - São Paulo (SP), Brazil.

Instituto Lauro de Souza Lima (ILSL) - Bauru (SP), Brazil.

Universidade de São Paulo (USP) - São Paulo (SP), Brazil.

(C)2015 by Anais Brasileiros de Dermatologia 
more appropriate, such as the detection rate of new cases, the detection rate of new cases with disability grade (DG) 2 at diagnosis, the treatment completion rate and the detection rate in people under 15 years of age. ${ }^{1,6}$

In Brazil, the rate of overall detection in 2011 was 17.65/100,000; and detection rate in people under 15 years of age was 5.22/100,000. This rate is considered very high and indicative of recent illness and of active transmission foci. The rate of DG 2 at diagnosis in 2011 was 11.3/100,000, which is also considered a high rate. The percentage of contacts investigated during this period was $59.8 \%$, which is considered average. These values confirm that even with a prevalence of 1,54:10,000 inhabitants in 2011, which is near the value established by the WHO and the Ministry of Health, leprosy is still an important public health problem in Brazil. ${ }^{1,7}$

M leprae transmission is not fully understood, especially due to the inability to cultivate it. However, trasmission seems to occur through aspiration aerosols of nasal secretion from active bacillipherous patients, in environments that concentrate large numbers of people under unfavorable socioeconomic conditions. ${ }^{1,5}$ The incubation period is 4 years, on average, for tuberculoid leprosy and 10 years for virchowian leprosy. ${ }^{8}$

The clinical features of the disease are determined by the host immune response to the bacillus. Nevertheless, little is known about the factors that lead to illness. ${ }^{1}$ Most infected people will not develop the disease, and $95 \%$ of individuals in the general population are resistant to $\mathrm{M}$. Leprae. ${ }^{4}$ After entering the organism, if the parasite is not destroyed, it will invade Schwann cells and subsequently be phagocytosed by skin macrophages. ${ }^{5}$ For this reason, patients whose infections develop to disease commonly present with skin lesions, as well as numbness and weaknessm wich are caused by the involvement of peripheral nerves. More rarely, patients may have a burning sensation in the lesions, in addition to trophic ulcers on their hands and anesthetic feet. Initial skin lesions (indeterminate leprosy) are generally poorly defined, xerotic or hypopigmented macules with still unchanged tactile sensitivity. ${ }^{2}$

From the initial injury, when the disease may spontaneously regress, leprosy usually evolves to one of the polar forms or to the unstable, dimorphic form. At one pole, there is the tuberculoid leprosy, in which patients have effective cellular immune response against the bacillus, which limits the disease to a few, well-defined anesthetic skin lesions, usually without involvement of nerve trunks. ${ }^{2,8,6}$

At the other pole, there is the so-called virchowian leprosy, in which patients have no specific cellular response to M. leprae. This allows for a slow, asymptomatic and uncontrolled proliferation of the bacilli. This spread is exteriorized in the form of diffuse dermal infiltrate, which leads to the "leonine aspect" when it reaches the face. Ankle edema, acrocyanosis, specific joint pain, loss of eyebrows and eyelashes, xerosis with ichthyosiform aspect may also occur. $^{2}$

According to Ridley (1974), however, most patients progress to intermediate and unstable forms of the disease, i.e., dimorphic forms. ${ }^{2,9}$

Neural involvement of leprosy affects all forms of the disease, from cutaneous nerve filaments - in the indeterminate form - to the involvement of nerves in the MB or PB forms. Nerve lesions that are less than 6 months old are considered premature, and can be reversed with corticosteroids, whereas lesions that are older than 6 months are considered to be difficult to revert. ${ }^{10}$ The longer evolution of the disease results in more intense and extensive neural lesion; the presence of disability at diagnosis represents worse prognosis for the occurrence of disabilities after PCT is finished. ${ }^{10,11,12}$ Thus, DG is an indicator that allows indirect evaluation of the effectiveness of early detection and appropriate treatment of cases. Alves et al found 167 new cases recorded at the Lauro de Souza Lima Institute between 2001 and 2006. The average time from onset of symptoms to diagnosis was 12 months. At diagnosis, $34 \%$ of these patients already had DG 1, $26 \%$ had DG 2 and only $35 \%$ had no disability. In $5 \%$ of cases no satisfactory data for evaluation were found. Our study has confirmed the direct relationship between time to disease progression and DG. This high rate $(60 \%$ of patients with disabilities at diagnosis), indicates a high rate of concealed prevalence and late diagnosis, since most patients with leprosy have no disability at onset of the disease. Moreover, it is noteworthy that during this study in 2005, leprosy was considered eradicated as a public health problem in the state of Sao Paulo. Recently, the Leprosy Control Coordination of the State of São Paulo reported that there has been a $41 \%$ decrease in the detection of leprosy cases in the last 10 years. ${ }^{13}$

The classification of leprosy is important in order to determine the prognosis, infectiousness and proper treatment of the disease. The methods of classification of leprosy have significantly changed over the years. Two methods are currently used: the Ridley and Jopling (R\&J) criteria, which is based on clinical, histopathological, immunological, developmental and sputum smear characteristics; and the WHO criteria, which is based only on the number of lesions.

This simplified WHO classification was proposed in 1995 due to the local unavailability of tests required by the R\&J criteria, such as histopathology, 
Mitsuda reaction, and skin smear microscopy. ${ }^{14}$

The WHO classifies patients as MB, when more than five lesions are present; or as paucibacillar (PB) when five or less lesions are present. ${ }^{2}$ According to the WHO classification, patients with virchowian leprosy present only diffuse infiltration. Primary neural and initial dimorphic forms with fewer visible lesions may be incorrectly classified, and therefore receive incorrect treatments. ${ }^{14}$

According to the R\&J criteria, the different forms of the disease correlate with the activity of the host's immune response and with his bacillar load. Based on this criteria, patients are divided into 6 categories: Indeterminate (I) or early diease, tuberculoid (TT), dimorphic tuberculoid (DT), dimorphic dimorphic (DD), dimorphic virchowian (DV) and virchowian (VV). Dimorphic forms are characterized by a gradual progression trend from DT to DV (downgrading), with increased bacillar load, increased antibody titer and increase in the number of skin and neural lesions. ${ }^{2,8}$

The bacillar load of a leprosy patient correlates with the presence of IgM antibodies against the phenolic glycolipid (PGL-1), unique to M. Leprae., , $, 11,12^{-12}$ Although leprosy diagnosis is clinical, as instructedby the $\mathrm{WHO}, 30 \%$ of patients do not present skin lesions with abnormal sensibility., ${ }^{4,14}$ The ELISA is a laboratory test that can be performed to aid in the diagnosis of leprosy in cases such as the one just described. It is used for the detection of anti-PGL-1 antibodies, found in approximately $78 \%$ of $\mathrm{MB}$ patients and $23 \%$ of $\mathrm{PB}$ patients. ${ }^{4}$ With treatment and fall of the bacillar load of the patient, there is a reduction of the titres of the antibody, which allows its use to help monitor the treatment. Increased titration values after the end of the treatment may be a clue to the early diagnosis of recurrence. ${ }^{3}$ IgM anti-PGL-1 serology also allows the easy identification of contacts with high risk of developing leprosy. ${ }^{4,11}$

Leprosy reactions are the major complications of leprosy and may occur before, during or after treatment. They usually leave sequelae. ${ }^{1,4,15}$ Moreover, they are common causes of inadequate retreatment, due to confusion with recurrence, in addition to being responsible for disability and treatment abandonment. ${ }^{4}$ Reactions are directly related to bacillar load and host immune response, and may be classified into two types: type- 1 reaction or reversal reaction (RT1) and type-2 reaction or erythema nodosum leprosum (ENL). ${ }^{9,15}$

RT1 is associated with the abrupt increase of cell mediated immunity; this affects more than half of the individuals and is the leading cause of neural damage in leprosy. It usually occurs during treatment, although it may be the initial presentation of the dis- ease. ENL comprises systemic inflammatory reaction related to the deposition of immune complexes; it occurs in approximately $50 \%$ of individuals with virchowian leprosy, and although it may be the first visible manifestation of the disease, it often appears after treatment. ${ }^{9,15,16}$

\section{OBJECTIVES}

The objectives of this study were to assess the social, clinical and laboratorial profile of patients who received a diagnosis of leprosy in the Lauro de Souza Lima Institute between January 2007 and December 2011.

Through this evaluation, we studied the variables that correlate with the early or late diagnosis of the diease, which helps to understand the true epidemiological situation of leprosy in the state of São Paulo.

\section{MATERIALS AND METHODS}

This study was descriptive and retrospective. We selected all biopsies with diagnostic suspecion of leprosy registered in the period from January 1, 2007 until December 31, 2011 and derived from the laboratory of pathological anatomy of the reference center Lauro de Souza Lima Institute. This institute serves patients from the public health system (SUS). From the biopsy reports, we extracted the medical records for data collection and selected only new cases of leprosy. The following data were collected and analyzed:

1) gender.

2) age at diagnosis

3) marital status.

4) educational level.

5) place of birth and residency.

6) whether the patient was referred to our facility with a diagnosis of leprosy or not.

7) whether the patient was summoned by the service for being a contact of a leprosy patient.

8) duraton of illness until diagnosis (in months).

9) clinical classification according to WHO criteria.

10) clinical classification according to Ridley and Jopling criteria.

11) anti-PGL-1 titration by ELISA

12) degree of disability at diagnosis presented by patients who underwent sensitivity testing with monofilaments.

The Kruskal-Wallis test was used to compare the duration of symptoms and the degree of disability found.

Chi-square test was used to evaluate the association between the classification and PGL-1.

Kappa statistics was used to compare the agreement between the WHO and the R\&J classifications.

A 5\% significance level was adopted for all sta- 
tistical tests $(p<0,05)$. All statistical procedures were conducted on Statistica software version 11 (StatSoft Inc., Tulsa, USA).

\section{RESULTS}

174 new cases of leprosy were diagnosed during the studied period, 71 (41\%) in women and 103 $(59 \%)$ in men. The mean age at diagnosis was 49 years, ranging from 6 to 86 years, with a standard deviation of $18.4(2.2 \%)$ cases were detected in children under 15 years of age.

90 patients $(51.7 \%)$ lived in the region where the service is located and 167 (96\%) patients were born in the State of São Paulo (Figure 1).

As for the educational level of patients, we found that $18(10 \%)$ patients were illiterate, $103(59 \%)$ had incomplete primary education, $11(6 \%)$ had complete primary education, $10(6 \%)$ had incomplete high school education, 25 (14\%) had complete high school education and $7(4 \%)$ had higher education degrees (Graph 1).

Regarding marital status, 88 (51\%) were married, $56(32 \%)$ were single, $17(10 \%)$ were divorced, and $13(7 \%)$ were widowed.

The average time from onset of symptoms to diagnosis was described in the record of $153(88 \%)$ patients. This time ranged from 15 days to 20 years, with a mean of 24 months and a standard deviation of 32; and with a median of 12 months and 25 percentile $=6$ and 75 percentile $=24$.

According to the R\&J criteria, 108 (62\%) cases were characterized as $\mathrm{MB}$ (dimorphic and virchowian); 65 (37\%) cases were classifed as PB (tuberculoid and indeterminate) and 1 case was not classified because data were missing in the medical record.

According to the WHO criteria, $113(65 \%)$ cases were $\mathrm{MB}, 50(29 \%)$ were $\mathrm{PB}$ and $11(6 \%)$ could not be

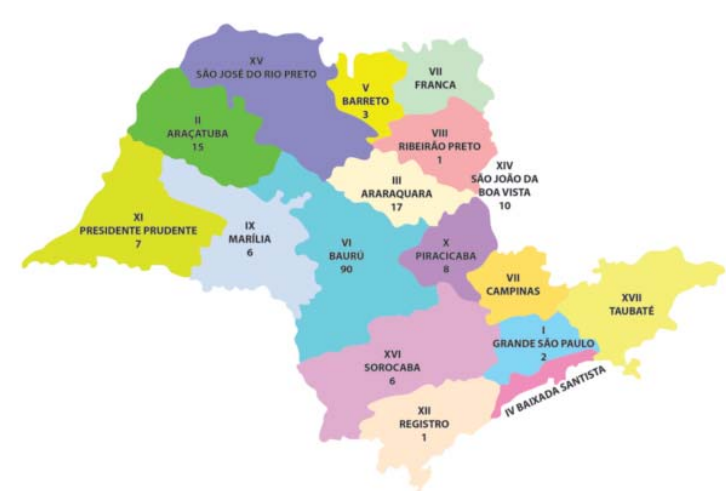

FIGURE 1: Distribution of patients from the state of São Paulo according to RHDs (Regional Health Departments)
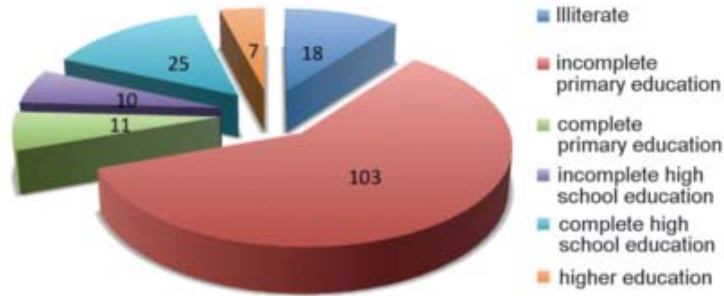

GrAPH 1: Educational level of patients diagnosed with leprosy in the Lauro de Souza Lima Institute in absolute numbers

classified because the number of lesions was not described in the medical records. Agreement between the two classifications was moderate $(74.8 \%)$, with kappa index $=0.44$.

Of the 174 diagnosed patients, 23 (13.2\%) were actively summoned for evaluation because they are contacts of patients being treated in the Lauro de Souza Lima Institute and because they had not been previously summoned at the primary care unit of origin . Among the latter, the average age was 40 years and no DG 2 was detected. The remaining 151 patients were referred to the Lauro de Souza Lima Institute. 74 (49\%) had suspicion of leprosy. For 77 (51\%) of these patients the diagnostic hypothesis of leprosy was not raised.

In $25(14 \%)$ cases, manifestations of leprosy reactions clinically evident at diagnosis were reported in the medical record. With regard to histopathology, $38(22 \%)$ of 172 patients who underwent this examination showed signs of leprosy reaction in the histopathological report.

DG at diagnosis was defined in 129 (74\%) of 174 patients diagnosed during the study period, but there were no data about this evaluation in $45(26 \%)$ records. Of 129 cases with DG described at diagnosis, $34(26 \%)$ presented grade $2,41(32 \%)$ grade 1 and 54 $(42 \%)$ grade zero (Table 1$)$. In graph 2 , we can see that the percentage of patients with DG zero fell from $50 \%$ to $35 \%$ over the five years of the study. There was no correlation between the time of onset of symptoms and DG, when applying the Kruskal-Wallis ANOVA test $(\mathrm{p}=0.6647)$.

TABLE 1: Absolute number of patients assessed for disability grade at diagnosis per year

\begin{tabular}{lllllll}
\hline DG & $\mathbf{2 0 0 7}$ & $\mathbf{2 0 0 8}$ & $\mathbf{2 0 0 9}$ & $\mathbf{2 0 1 0}$ & $\mathbf{2 0 1 1}$ & TOTAL \\
\hline 0 & 7 & 18 & 9 & 11 & 9 & 54 \\
1 & 1 & 9 & 8 & 13 & 10 & 41 \\
2 & 6 & 11 & 4 & 6 & 7 & 34 \\
\hline TOTAL & $\mathbf{1 4}$ & $\mathbf{3 8}$ & $\mathbf{2 1}$ & $\mathbf{3 0}$ & $\mathbf{2 6}$ & $\mathbf{1 2 9}$ \\
\hline
\end{tabular}


Anti- PGL-1 serology was performed in 87 cases. All 87 cases were classified according to R\&J and 79 (out of 87) were classified according to the WHO. A positive serology was statistically correlated with multibacillary classification, and negative serology with paucibacillary classification, both by using R\&J criteria and the simplified WHO system. The chisquare test showed statistical significance $(p=<0.001)$ for the association between R\&J criteria and PGL-1; and also for the association between WHO criteria and PGL-1 $(p=0.008)$ (Table 2).

\section{DISCUSSION}

Leprosy is a disease that predominantly affects males, possibly because men are more exposed to the bacillus in non-domestic environments. This study demonstrated an involvement ratio of 1.45 men for 1 woman, and is consistent with the data found in the literature. ${ }^{5}$ Our findings do not diverge from the data found in the rest of the country: the economically lessfavored population, with lower educational levels is more affected. ${ }^{14} 121$ (70\%) patients diagnosed with leprosy in this study were illiterate or only had incomplete primary education. This variable alone is already a factor that would justify patient delay in seeking diagnosis.

About half of the patients who made up the new cases were married (a), and these patients were referred to the service without suspicion of leprosy, i.e., together with/among the general dermatological demand. The fact that new cases were found and diagnosed only after their relatives had been summoned to come to the Institute indicates a serious epidemiological surveillance failure in the state of São Paulo, and the lack of training of primary care professionals with regard to leprosy. The average age of patients was around 50 years, and most of them had low educational levels, which supports the need to maintain surveillance activities in the state, since these data do not differ from the reality found in the rest of the country. ${ }^{14}$
The economically active group of patients diagnosed (mean age $=50$ years) may have negative repercussions for the economy of the state, since this population may ultimately develop disabilities, reactional states and have to be prematurely withdrawn from their productive activities. Besides, this could generate high social costs. Onset of disease among this age group was also not supposed to be found, since leprosy was eliminated as a public health problem in São Paulo 7 years ago. It is reported in the work of et al that, in areas of low endemicity (low load of bacilli in the environment), only the most susceptible people would get sick, i.e., elderly virchowian patients, which was not observed in this study. The hypothesis of a migration of individuals already ill who were subsequently diagnosed in the state of São Paulo is also ruled out, as $96 \%$ of cases were born in the state of São Paulo, and 90 cases $(51.7 \%)$ lived in the São Paulo, indicating that the endemic is autochthonous.

Because of the long incubation period of leprosy, household contacts of patients, also called contacts, are a risk group up to 10 times more likely to acquire the disease. Although it is important to maintain the epidemiological chain of leprosy, little effort has been directed to the control of the disease in this group..$^{10}$ Proper investigation of contacts allows early diagnosis and interrupts the chain of transmission. The examination of contacts should not be a routine of the referral service, but rather of the basic health unit (BHU). However, diagnosed patients coming to the rheumatology outpatient clinic where the study was conducted due to appointments or treatment of complications frequently report that their relatives were only vaccinated at the BHU but not examined. Due to this fact, some contacts are actively summoned to the clinic for examination.

An estimated 3 million people who have completed treatment with PCT have irreversible physical disabilities as a result of neural involvement in leprosy. These patients need continuous care in order to prevent higher and secondary damage to the changes already present. ${ }^{5}$

TABLE 2: Association between anti-PGL-1 serology, and the WHO and the R\&J classifications

\begin{tabular}{lllllll}
\hline SEROLOGY & $\begin{array}{l}\text { WHO } \\
\text { Positive } \\
\text { Anti-PGL-1 }\end{array}$ & $\begin{array}{l}\text { Negative } \\
\text { Anti-PGL-1 }\end{array}$ & Total & $\begin{array}{l}\text { R\&J } \\
\text { Positive } \\
\text { Anti-PGL-1 }\end{array}$ & $\begin{array}{l}\text { Negative } \\
\text { Anti-PGL-1 }\end{array}$ & Total \\
MB (n; $\%)$ & $\begin{array}{l}29(55 \%) \\
\text { PB (n; } \%)\end{array}$ & $24(45 \%)$ & 53 & $35(64 \%)$ & $20(36 \%)$ & 55 \\
\hline TOTAL & 35 & $20(77 \%)$ & 26 & $4(12 \%)$ & $28(87 \%)$ & 32 \\
\hline
\end{tabular}

WHO classification $\times$ PGL-1: $p=.008$ R \& J Classification $\times$ PGL-1: $p=<.001$ 
Disability is defined as an anatomical or physiological change, which prevents or hinders patients from performing an activity or having a normal social interaction for their age, cultural pattern, income and educational level. If not diagnosed and treated early, leprosy can evolve with different DG. ${ }^{1,3}$ Thus, this indicator allows an indirect assessment of the effectiveness of early detection and proper treatment of leprosy cases. ${ }^{10}$ According to the Ministry of Health (MS), the DG assessment should be performed at diagnosis, at least once a year during treatment, and at the end of treatment. ${ }^{3}$ The MS states that in 2011, the DG was assessed in $89.5 \%$ of diagnosed cases. This percentage is superior to the average found between 2007 and 2011 in the reference center where this study was conducted $(74 \%) .{ }^{7}$ The DG 2 found in this study is well above the national average. This grade also diverge from the data released by the State. In 2010 in the state of São Paulo $9.8 \%$ of patients had DG 2 at diagnosis; in 2011, the MS describes $11.3 \%$ of diagnosed patients with DG 2. ${ }^{7}$ Mean DG 2 during the studied period was $26 \%$ in the reference service where the study was conducted. Since it is a referral center, the higher percentage of DG 2 at diagnosis could be justified because more complicated cases, i.e., difficult to clinically diagnose, are referred to the institute. Nevertheless, less than half of cases had at least a diagnostic suspicion when they were referred.

Another fact that allows us to question whether the dramatic fall in detection in the last 10 years really reflects control of the disease is the progressive reduction in the number of patients diagnosed without disabilities, as shown in graph 2 . The ineffectiveness of activities for early detection of leprosy and consequent late diagnosis justify this reduction in the percentage of patients with DG 0 at diagnosis.

Alves et al evaluated the DG of patients diagnosed at the same service investigated in this study between 2001 and 2006. They found DG zero in 35\% of patients, DG 1 in $34 \%$ and DG 2 in $26 \%$ of patients.

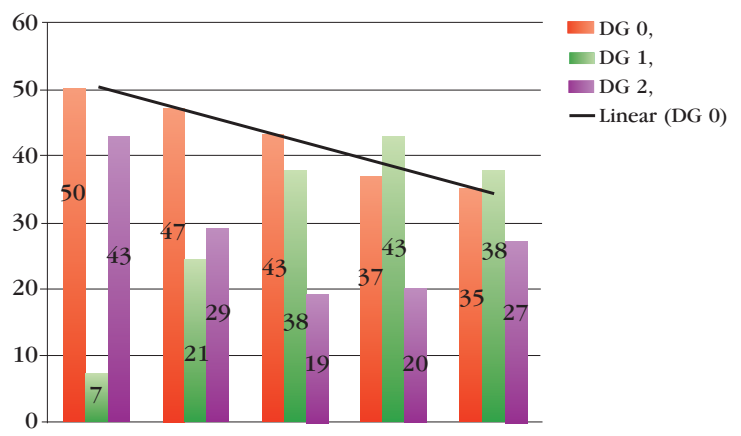

GRAPH 2: Percentage of disability grade at diagnosis per year
As for the period 2007-2011, we found DG zero in $42 \%$, DG 1 in $32 \%$ and DG 2 in $26 \%$ of patients; in $26 \%$ of cases there were no data on the assessment. ${ }^{3}$

Another finding by Alves et al was the average of 12 months of duration of symptoms to diagnosis. This average time doubled to 24 months for the period between 2007 and 2011. ${ }^{3}$

This fact indicates the unpreparedness of health professionals from primary care in the state of São Paulo to make diagnostic suspicion. Decentralization of care of patients with leprosy was not accompanied by an adequate training of health professionals. It is reported that the integration of leprosy care to the services provided at primary care has led to a loss of skill in the diagnosis and management of leprosy, ${ }^{1}$ since the diagnosis of this disease requires skill and enough time to perform the physical examination.

The correct classification of leprosy cases is an essential tool for understanding the disease. It also determines the prognosis, which individuals are infectious, and indicates which is the most adequate treatment. ${ }^{1,2,14,17,18}$ Due to the wide spectrum of clinical manifestations of the disease, the use of histopathological criteria, smear microscopy, evolutionary and immunological criteria, as proposed by the R\&J classification in 1966 allows greater sensitivity and specificity rates for disease classification and are widely accepted by pathologists and leprosy specialists. ${ }^{1,16,19,20}$ Complementary examinations, however, are usually not available in most BHUs, where the diagnosis and classification of the disease should be made. Due to this problem, the WHO proposed a simplified classification, using only clinical criteria. ${ }^{6,14,18,20,21,22}$ This classification has benefits and limitations. While it is easy and simple to application by general practitioners, there will always be the risk of under- or over-treatment. The sensitivity rate for the proper classification of leprosy using WHO criteria is described in most studies as 85\% to $93 \%$. However, most of these studies relied on smear microscopy as a gold standard, and this examination is characterized by high specificity but low sensitivity rates $(10-50 \%)$. Given the fact that many professionals do not collect material from lesions due to lack of practical training, the smear microscopy may be false-negative in MB patients, especially in those patients with few sublesions. ${ }^{22}$ The sub-treatment of patients with both $\mathrm{MB}$ and $\mathrm{PB}$ increases the risk of resistance, recurrence, and allows the maintenance of the chain of disease transmission. . $^{16,17,20,22}$

Although a moderate correlation between the WHO and the R\&J classification was found in this study, the first is not justified in references. In this study, we found that just over $5 \%$ of new cases would not be diagnosed only by means of an appropriate clinical evaluation. 
If only the WHO criteria had been used to classify patients, and using smear microscopy as the gold standard, $11 \%$ of patients in the study by Norman G. et al (2004) would have been under-treated, while 13\% would have been over-treated. ${ }^{17}$ In the study by Barreto et al (2008), just over a quarter of dimorphic patients had up to 5 skin lesions, similar to what was reported by Bhushan et al (2008), who observed that the WHO classification incorrectly classified 20 of 76 (26.3\%) MB patients as PB and 18 of 65 (27.69\%) PB patients as $\mathrm{MB}$, using histopathological analysis as the gold standard. ${ }^{18,22}$

In the study by Pardillo et al (2008), 38\% of patients with less than 5 skin lesions had histopathologic features of dimorphic forms, i.e., MB; and only $57 \%$ of those patients had smear microscopy of index points higher than or equal to one. This study concluded that, especially in areas of high frequency of MB patients, smear microscopy and anatomicopathological examination should be reintroduced for patient classification and diagnosis determination. ${ }^{20}$

Teixeira et al (2008) show a $67.6 \%$ clinical and laboratorial concordance in the diagnosis of leprosy and criticize the use of purely clinical classifications of leprosy for referral centers, education and research. ${ }^{16}$ Unfortunately, many health professionals working in basic healthcare units believe that smear microscopy and biopsy should mandatory. This is due to feelings of insecurity and lack of training to make clinical diagnosis. For diagnosis of advanced cases, however, as in this series of cases, the WHO classification is sufficient.

This study also revealed that, according to the WHO classification, 50 patients would be classified as $\mathrm{PB}$ and 114 as MB; using the R\&J criteria, these values would be $65 \mathrm{BP}$ and $108 \mathrm{MB}$. In order to compare the two classifications, 10 patients had to be excluded, because the number of lesions was not registered on their medical records and 1 patient was excluded because there was not sufficient data on the records to perform a R\&J classification. The agreement rate found between the WHO and the R\&J classifications was $74.84 \%$, which is considered moderate, with kappa index $=0,44 .{ }^{23,24}$ The WHO classification, when diagnosis is late, is sufficient for the diagnosis of most cases (dimorphic), with great accuracy, sparing laboratory tests, which should be reserved for virchowian cases without visible plaques.

Leprosy diagnosis is essentially clinical and epidemiological. It is performed through the analysis of the history and living conditions, and the dermatological and neurological examination of the patient, in order to identify skin lesions or areas with abnormal sensitivity and/or involvement of peripheral nerves. ${ }^{4,14}$ However, $30 \%$ of patients, including several
MB patients, show no clinical manifestation of the disease, which hinders the diagnosis and the breaking of the transmission chain. ${ }^{4,6,14,25}$ Classifying these patients into $\mathrm{PB}$ and $\mathrm{MB}$ is very important, because treatment differs for both groups. Laboratory tests (histopathology, Mitsuda's reaction, smear skin microscopy and serology) may be used to assist in the correct classification of these cases. These, however, are not available at most of the health services. ${ }^{14}$

Measurement of the IgM serum antibody against PGL-1, assessed by ELISA, is considered a relevant disease activity marker of leprosy. Phenolic glycolipid-1 (PGL-1) is a cell-wall antigen on the surface of $M$. leprae and stimulates the production of specific immune response in patients. ${ }^{4,14}$ Antibody levels are related to the severity of the disease and the bacillar load of the patient. PGL-1 positivity cannot be used as an isolated diagnostic criterion, but can be used in the diagnostic process, when serological results are considered together with other clinical data and diagnoses. Among contacts of leprosy patients, PGL-1 positivity increases eight times the risk of developing the disease. ${ }^{11,12,14}$

In the present study, anti-PGL-1 positivity was correlated with $\mathrm{MB}$ disease, with statistical significance, regardless if the classification used is the WHO or the R\&J. However, we found a greater difference to the R\&J classification. This fact reveals a higher accuracy of the R\&J classification for more complicated cases, such as primary neural cases, dimorphic cases with few lesions and virchowian, virtually asymptomatic cases.

Leprosy reactions are may occur before, during or after treatment with PCT. They immensely contribute to the disabilities of leprosy, and need to be diagnosed early in order to prevent loss of neural function and disability. More than half of patients with dimorphic leprosy will develop RT1, and half of these will evolve to loss of neural function. ENL affects around half of patients in the lepromatous spectrum, but has a less dramatic nervous involvement than RT $1 .{ }^{22}$ That is, over $50 \%$ of leprosy patients will present reaction episodes during the course of the disease. In the present study, clinical evaluation underestimated the presence of leprosy reaction in relation to histopathological diagnosis. This may result in potential risk of progression to disability.

Recently, the Leprosy Control Coordination of the State of São Paulo reported that there was a $41 \%$ decrease in the detection of leprosy cases over the last 10 years. ${ }^{13}$ This fall would be the result of an improvement in quality of care and decentralization of care of patients with leprosy. Thus, the diagnosis of 23 cases should not be expected, only because they were actively summoned for evaluation, nor the finding of 
so many cases with an installed DG. It is important to highlight that, among patients actively summoned due to referral, unlike patients spontaneously diagnosed, the diagnosis was significantly earlier (on average, a decade earlier). $80 \%$ of the patients assessed in this group had DG zero, 20\% had DG 1, and none had DG 2. These data confirm that early diagnosis, even before patients seek medical care, is essential in the prevention of disabilities.

In this study, we observed a decrease in the rate of patients assessed for DG, even when they had been diagnosed at referral. This fact, added to the increase in the time between the onset of symptoms and diagnosis, and to the progressive fall in the rate of DG zero at diagnosis, serves as a warning to the State's health surveillance system. DG at diagnosis should be considered if rated up to 1 month after diagnosis, and although it is a quick and simple assessment, it is not always performed.

Habbema, apud Rodrigues \& Lockwood, argues that, for the eradication of an infectious disease, it is necessary an intervention that make it possible to break the transmission chain. ${ }^{1}$ In addition, there should also be practical diagnostic tools, with sufficient sensitivity and specificity to detect all levels of infection. Based on these principles, he suggests that extensive epidemiological and microbiological investigation should be made to enable the understanding of the magnitude of the disease at a certain site, as well as the development of diagnostic tools for the early detection of the infection. New interventions, such as vaccination and chemoprophylaxis should also be developed and implemented. ${ }^{1}$

\section{CONCLUSION}

In the present study, male patients with low educational levels, born in the state of São Paulo, and in the economically active age group were predominantly affected, which results in large economic losses.

Although half of them are married, diagnosis of new cases ocurred after active summoning of relatives, which indicates the lack of training or neglect of health professionals of basic healthcare units.

The mean duration of symptoms to diagnosis doubled when the predominantly pre- and post-elimination periods were compared. The rates of disability grade at diagnosis and lack of suspicion remained high.

About $20 \%$ of cases were only referred to the service when they evolved with leprosy reaction. This indicates that patients spontaneously seek for medical care and there is a lack of training of local health professionals to perform the diagnosis, as well as a lack of contacts surveillance.

When an active search for contacts was performed, the profile of new diagnosed cases changed completely, which indicates the need for such action.

There was moderate agreement between the WHO and the R\&J classifications. The first is useful in field practice, but when used alone, it proved to be insufficient for classification in 5\% of cases.

The data collected in this study raise doubts whether the disease is truly eradicated in São Paulo.

More studies are needed to help understand the true epidemiological situation of the disease in the state of São Paulo, since this expressive fall in detection could be purely due to operational reasons.

The active search for patients, as well as the supervision and training of primary care services will be the initial steps towards this goal (control). These measures will be needed for decades before leprosy can be considered a disease of the past. 


\section{REFERENCES}

1. Rodrigues LC, Lockwood DNJ. Leprosy now: epidemiology, progress, challenges, and research gaps. Lancet Infect Dis. 2011;11:464-70.

2. Walker SL, Lockwood DN. Leprosy. Clin Dermatol. 2007;25:165-72.

3. Alves CJ, Barreto JA, Fogagnolo L, Contin LA, Nassif PW.. Evaluation of the degree of incapacity of patients with a diagnosis of leprosy at a dermatology service in the state of Sao Paulo. Rev Soc Bras Med Trop. 2010:43:460-1.

4. Moura RS, Calado KL, Oliveira ML, Bührer-Sékula S. Leprosy serology using PGL-I: a systematic review. Rev Soc Bras Med Trop. 2008:41:11-8.

5. Britton WJ, Lockwood DN. Leprosy. Lancet. 2004;363:1209-19.

6. Barreto JA. Avaliação de pacientes com hanseníase na faixa virchowiana diagnosticados entre 1990 e 2000 e tratados com poliquimioterapia 24 doses e seus comunicantes na fase pós-eliminação em municípios de Santa Catarina. São Paulo: Universidade de São Paulo; 2011.

7. Portal.saude.gov [Internet]. Ministério da Saúde. Coeficientes de detecção geral de casos novos de hanseníase Brasil e estados. [acesso 8 Ago 2012]. Disponível em: http://www. portal.saude.gov.br

8. Ridley DS, Jopling WS. A classification of leprosy for research purposes. Lepr Rev. 1962;33:119-28.

9. Mendonça VA, Costa RD, Brito-Melo GE, Antunes CM, Teixeira AL. Immunology of leprosy. An Bras Dermatol. 2008;83:343-50.

10. Pimentel MIF, Nery JAC, Borges E, Gonçalves RR, Samo EN. Influência do tempo de evolução prévio ao diagnóstico inicial incapacidades presentes no exame inicial de pacientes portadores de hanseníase multibacilar. Hansen Int. 2002;27:77-82.

11. Bazan-Furini R1, Motta AC, Simão JC, Tarquínio DC, Marques W Jr, Barbosa MH, et al. Early detection of leprosy by examination of household contacts, determination of serum anti-PGL-1 antibodies and consanguinity. Mem Inst Oswaldo Cruz. 2011;106:536-40.

12. Sinha S, Kannan S, Nagaraju B, Sengupta U, Gupte MD. Utility of serodiagnostic tests for leprosy: a study in an endemic population in South India. Lepr Rev. 2004;75:266-73.

13. Saude.sp.gov.br [Internet]. Em 10 anos, novos casos de hanseníase caem $41 \%$ em SP. [acesso 30 jul 2012]. Disponível em: http:// www. saude.sp.gov.br/ ses/noticias/2012/agosto/em-10-anos-novos casos-de-hanseniase-caem-41-em-sp

14. Contin LA, Alves CJ, Fogagnolo L, Nassif PW, Barreto JA, Lauris JR, et al. ML-Flow test as a tool in classifying and treating leprosy. An Bras Dermatol. 2011;86:91-5.

15. Kahawita IP, Walker SL, Lockwood DNJ. Leprosy type 1 reactions and erythema nodosum leprosum. An Bras Dermatol. 2008;83:75-82.

16. Teixeira AC, Cruvinel DL, Roma FR, Luppino LF, Resende LH, Sousa Td, et al. Evaluation of the agreement between clinical and laboratorial exams in the diagnosis of leprosy. Rev Soc Bras Med Trop. 2008;41:48-55.
17. Norman G, Joseph G, Richard J. Validity of the WHO operational classification and value of other clinical signs in the classification of leprosy. Int J Lepr Other Mycobact Dis. 2004;72:278-83.

18. Barreto JA, Nogueira ME, Diorio SM, Bührer-Sékula S.. Leprosy serology (ML Flow test) in borderline leprosy patients classified as paucibacillary by counting cutaneous lesions: an useful tool. Rev Soc Bras Med Trop. 2012;41:45-7.

19. Panday AN, Tailor HJ. Clinocohistopathological correlation of leprosy. Indian J Dermatol Venereol Leprol. 2008;74:174-6.

20. Pardillo FE, Fajardo TT, Abalos RM, Scollard D, Gelber RH. Methods for the classification of leprosy for treatment purposes. Clin Infect Dis. 2007;44:1096-9.

21. Mehndiratta RC, Patnaik A, John 0, Rao PS. Does nerve examination improve diagnostic efficacy of the WHO classification of leprosy. Indian J Dermatol Venereol Leprol. 2008;74:327-30.

22. Bhushan P, Sardana K, Koranne RV, Choudhary M, Manjul P. Diagnosing multibacillary leprosy: a comparative evaluation of diagnostic accuracy of slit-skin smear, bacterial index of granuloma and WHO operational classification. Indian J Dermatol Venereol Leprol. 2008;74:322-6.

23. Fleiss JL. Statistical methods for rates and proportions. New York: John Wiley \& Sons; 1973.

24. Landis JR, Koch GG. The measurement of observer agreement for categorical data Biometrics. 1977;33:159-74.

25. Oliveira ML, Cavaliére FA, Maceira JM, Bührer-Sékula S. The use of serology as an additional tool to support diagnosis of difficult multibacillary leprosy cases: lessons from clinical care. Rev Soc Bras Med Trop. 2008;41:27-33.

\author{
MAILING ADDRESS: \\ Ana Carolina Souza Porto \\ Rua Loefgreen, 1654, apto 71 - Vila Clementino \\ 04040-002 - São Paulo - SP \\ Brazil \\ E-mail:carolsporto1@hotmail.com
}

How to cite this article: Porto ACS, Figueira BFCF, Barreto JA, Lauris JRP. Evaluation of the social, clinical and laboratorial profile of patients diagnosed with leprosy in a reference center in São Paulo. An Bras Dermatol. 2015;90(2):169-77. 